\title{
Tilt Angle Measurement Based on Arrayed Eddy Current Sensors
}

\author{
Xuewei Chao ${ }^{1,2}$, Yang $\mathrm{Li}^{1 *}$, and Jing $\mathrm{Nie}^{1}$ \\ ${ }^{1}$ College of Mechanical and Electrical Engineering, Shihezi University, Shihezi, Xinjiang 832003, China \\ ${ }^{2}$ Department of Electrical and Electronics Engineering, Dalian University of Technology, Dalian, Liaoning 116024, China
}

(Received 8 November 2016, Received in final form 8 December 2016, Accepted 8 December 2016)

\begin{abstract}
Eddy current (EC) sensor works based on the electromagnetic induction principle and has been widely applied in the industrial testing and evaluation due to its robustness and environmental adaptability. Meanwhile, tilt angle measurement is mainly based on the laser or visual method, which is strict with the measurement environment and not suitable for the industrial applications. In this paper, a novel tilt angle measurement method based on arrayed EC sensors is proposed. Both the simulation and experiments indicate that the measured error is approximately linear with tilt angle and the accuracy after compensation is $0.25^{\circ}$. In conclusion, this research cannot only broaden the scope of EC application, but also overcome the shortcomings of existing angle measurement methods.
\end{abstract}

Keywords : eddy current, tilt angle, electromagnetic, measurement, inclined plate

\section{Introduction}

Eddy current (EC) sensor is working based on the electromagnetic induction principle and now has been widely applied in the field of displacement measurement [1-3], defect detection [4-6], electric conductivity measurement and many other fields [7-9], owing to its noncontact, no pollution, high sensitivity and robustness.

Although the EC sensors have many applications, the related research associated with tilt angle is still few. So the tilt angle measurement based on arrayed EC sensors may be very meaningful. At present, the tilt angle measurement is mostly through the visual and laser methods $[10,11]$, which are very strict with the measurement environment, which means these methods are not suitable for the industrial environment. However, the EC sensor is very suitable for industrial measurement, owning to its strong environmental adaptability. Hence, the combination of tilt angle measurement and EC testing may be interesting, which not only makes up for the shortcomings of the existing methods, but also broadens the scope of the EC applications.

In this paper, a novel application based on the EC method is proposed. In order to testify its feasibility, the

(C)The Korean Magnetics Society. All rights reserved.

*Corresponding author: Tel: +86-188-9959-7887

Fax: 0993-2057237, e-mail: liyang328@shzu.edu.cn simulation and experiments are carried out. According to the results, there is an approximately linear relationship between average measured error and tilt angle of the tested plate. After compensation, the largest error is $0.25^{\circ}$.

\section{Theoretical Analysis Model}

Three eddy current (EC) sensors were chosen to measure the tilt angle of inclined plate, the reason is that at least three points are needed to determine a plane. But, the magnetic coupling interference among the arrayed EC sensors must be taken into account when multiple EC sensors were working together.

It is obvious that the spacing between sensors has impact on the coupling interference and affects the measured result. When the spacing is equal to or larger than the coil's diameter, each sensor's output characteristics would not be affected [12]. So the spacing between sensors is set as the coil's diameter, by this means, the magnetic coupling interference could be neglected. The theoretical analysis model is shown as Fig. 1.

Where the point $\mathrm{A}, \mathrm{B}, \mathrm{C}$ represents the $\mathrm{EC}$ sensor and the point A', B', C' stands for the measured point on the tested plate. The two planes (ABC and $\left.\mathrm{A}^{\prime} \mathrm{B}^{\prime} \mathrm{C}^{\prime}\right)$ represent the reference plane and tested surface, respectively. And the vector $\mathrm{z}$ ' and $\mathrm{n}$ stands for the normal vector of the two planes, respectively. Thus, the tilt angle of inclined plate will be equal to the angle between the two vectors, which 


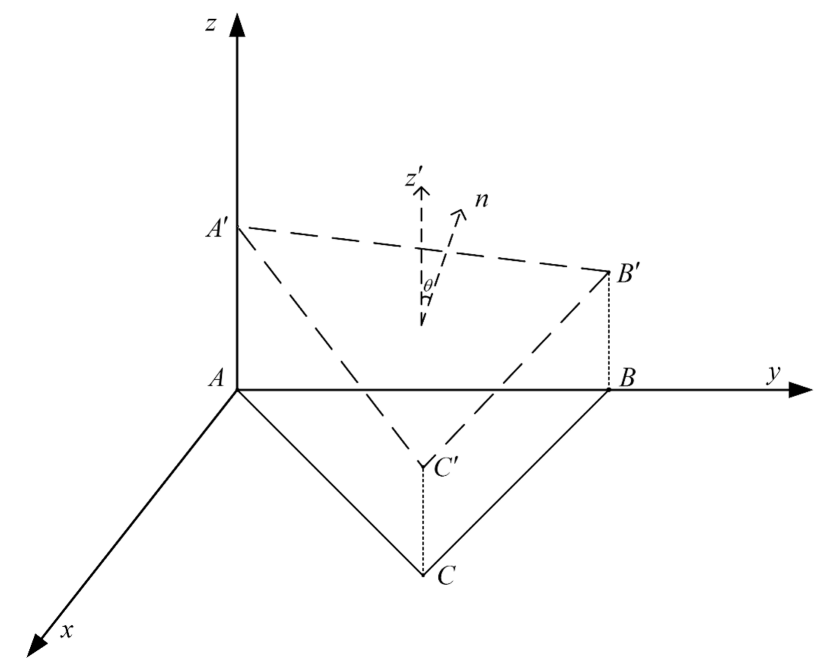

Fig. 1. The theoretical analysis model.

could be obtained by the vector operations and inverse trigonometric function calculations.

\section{Simulation Analysis}

All the finite element method (FEM) simulation analyses are carried out through the ANSYS Maxwell, which is an electromagnetic simulation software. The material of coil and tested plate is set as copper and aluminum, respectively. The inner, outer diameter and thickness of coil is 15,20 and $3 \mathrm{~mm}$. The tested plate is a flat sample with diameter $120 \mathrm{~mm}$ and thickness $10 \mathrm{~mm}$. Distribution of the three coils is an equilateral triangle and the side length is equal to outer diameter $20 \mathrm{~mm}$. Excitation frequency is set as $10 \mathrm{kHz}$ and the solution region is $300 \%$. Moreover, the mesh operation for the three coils and measured plate is set as inside subdivision and skin depth subdivision, respectively. Besides, the whole solution type should be selected for Eddy Current. The simulation model is shown as Fig. 2.

Due to the impact of tilt angle on EC displacement

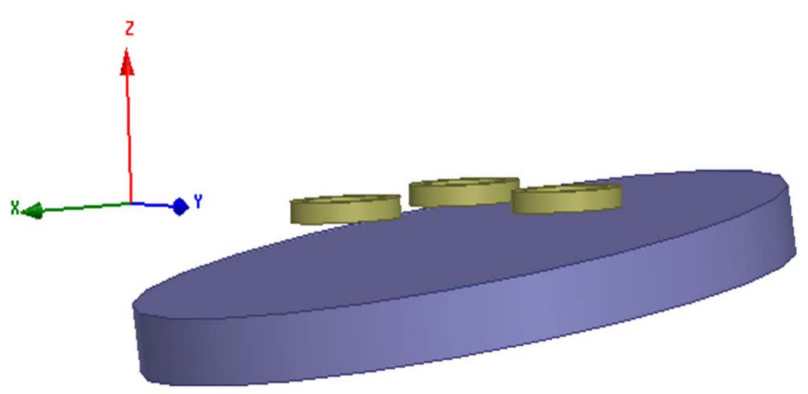

Fig. 2. (Color online) The established simulation model.

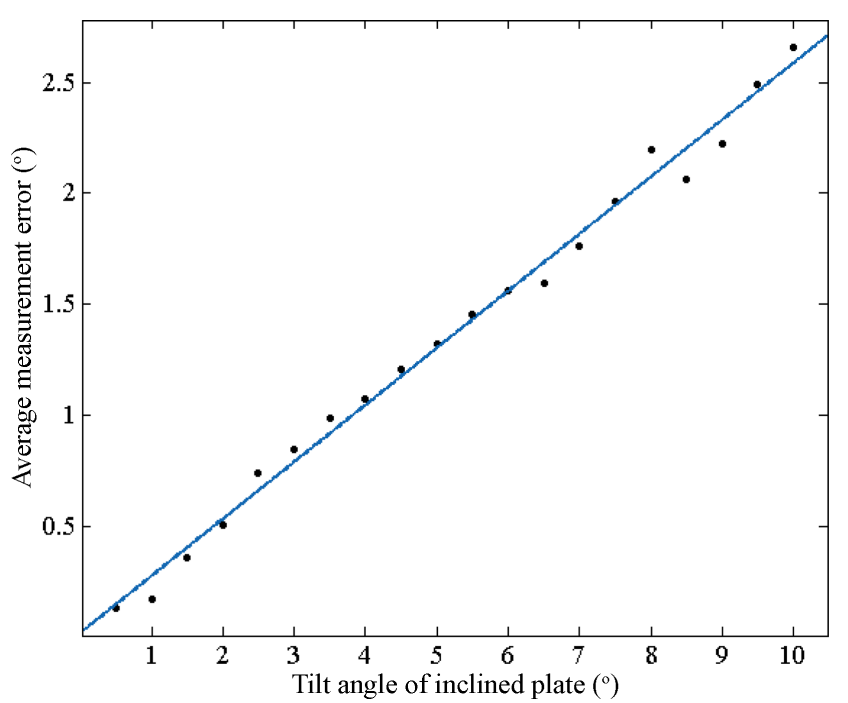

Fig. 3. (Color online) The relation between tilt angle and average error by simulation.

measurement [13], the measured error cannot be ignored. So, there must be a corresponding relationship between the final measured value and the tilt angle of inclined plate. In the simulation, the tilt angle of inclined plate is set from $0.5^{\circ}$ to $10^{\circ}$ and the interval is $0.5^{\circ}$. In order to make this research more sense, the operations were repeated three times under each condition. And the difference of measured values is very small. So, the average value of different measured error under certain tilt angle will be taken as the final measured error. The relationship between tilt angle and average measured error is shown as Fig. 3.

It can be discovered that the average measured error is approximately linear with the tilt angle of inclined plate,

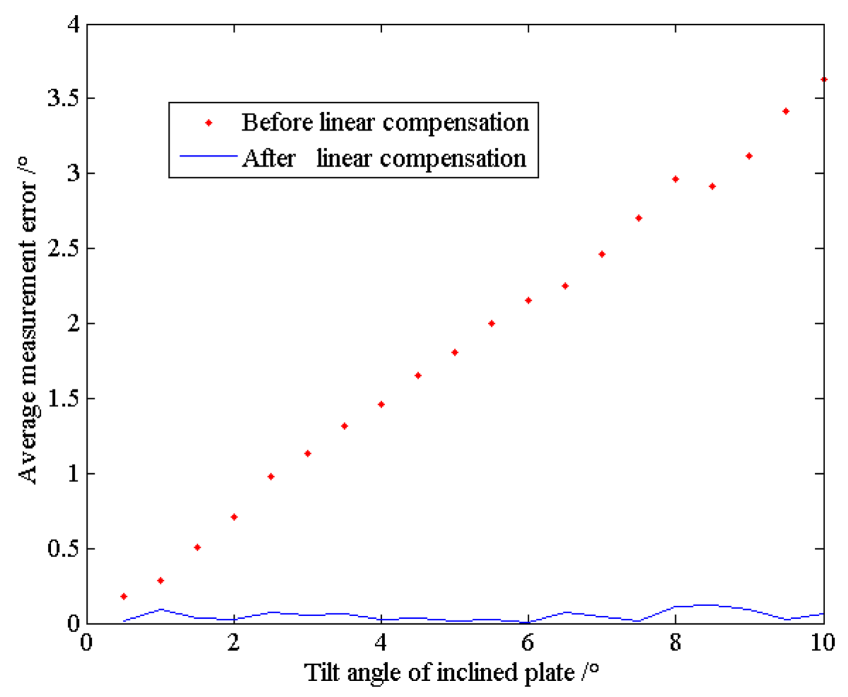

Fig. 4. (Color online) The measured error before and after compensation by simulation. 
which means the measured error could be compensated well by the linear compensation. In Fig. 3, the fitting linear function is as follows:

$$
f(x)=0.3556 * x+0.01555
$$

Where, the independent variable $x$ represents the tilt angle of inclined plate and the dependent variable $f(x)$ stands for the average measured error. The sum of squares due to error (SSE) is 0.0712 and the coefficient of determination $\left(\mathrm{R}^{2}\right)$ is 0.9966 .

Through the simulation, the measured error before and after compensation is shown in Fig. 4. It is shown that the largest error after compensation is $0.12^{\circ}$, which means the linear compensation could provide a good compensation accuracy.

\section{Experimental Analysis}

The above simulation analysis has revealed the linear relationship between the average measured error and tilt angle. In order to testify its correctness, multiple groups of experiments have been performed. The Japan KEYENCE EC sensor is selected, 6061 aluminium alloy plate is selected as the tested plate and the tiny displacement platform with dial indicator is selected as the experimental platform. Moreover, the platform has a rotation axis to rotate the tested plate. And the targeted tilt angle is obtained though a digital protractor, whose resolution is $0.1^{\circ}$. The experimental setup is shown as Fig. 5 .

Same as the theoretical analysis model, the arrayed EC sensors are arranged as Fig. 6. The rotation axis of the platform is shown in Fig. 7. Based on the digital protractor, any fixed tilt angle can be set by rotating the rotation axis of the platform. And the operation process is as follows: set the bench surface as reference $\left(0^{\circ}\right)$, measure tilt angle of tested plate, modify and tightly lock the plate, which is shown in Fig. 8.

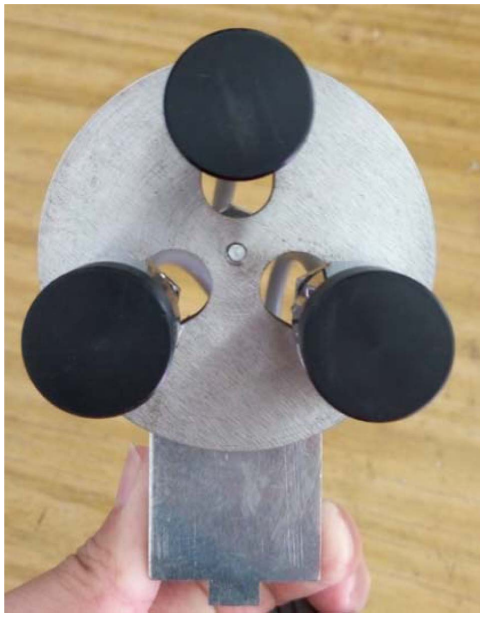

Fig. 6. (Color online) The arrayed EC sensors.

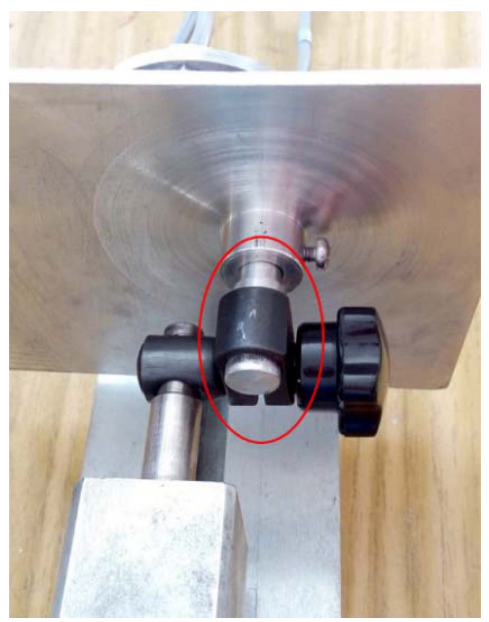

Fig. 7. (Color online) The rotation axis.

Similar to the simulation, the tilt angle of inclined plate varies from $1^{\circ}$ to $9^{\circ}$ and the interval is $1^{\circ}$. In order to test the repeatability, five different groups of experiments are carried out under each condition. The average measured

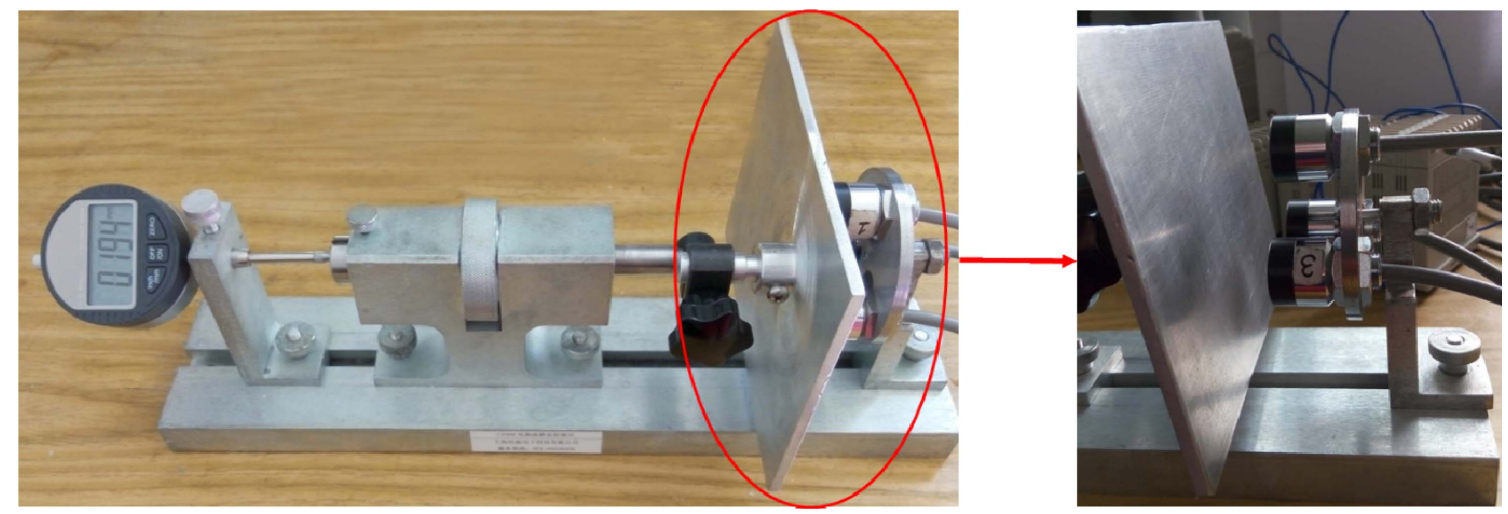

Fig. 5. (Color online) The whole experimental setup. 


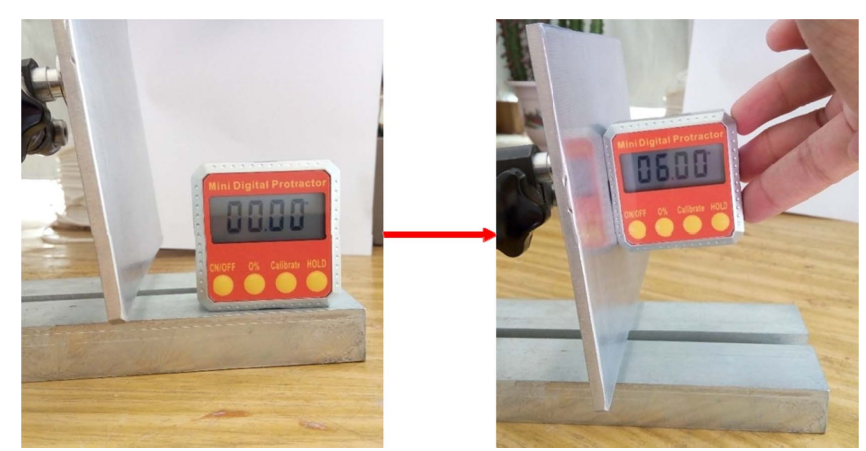

Fig. 8. (Color online) The operation process of the establishment of tilt angle.

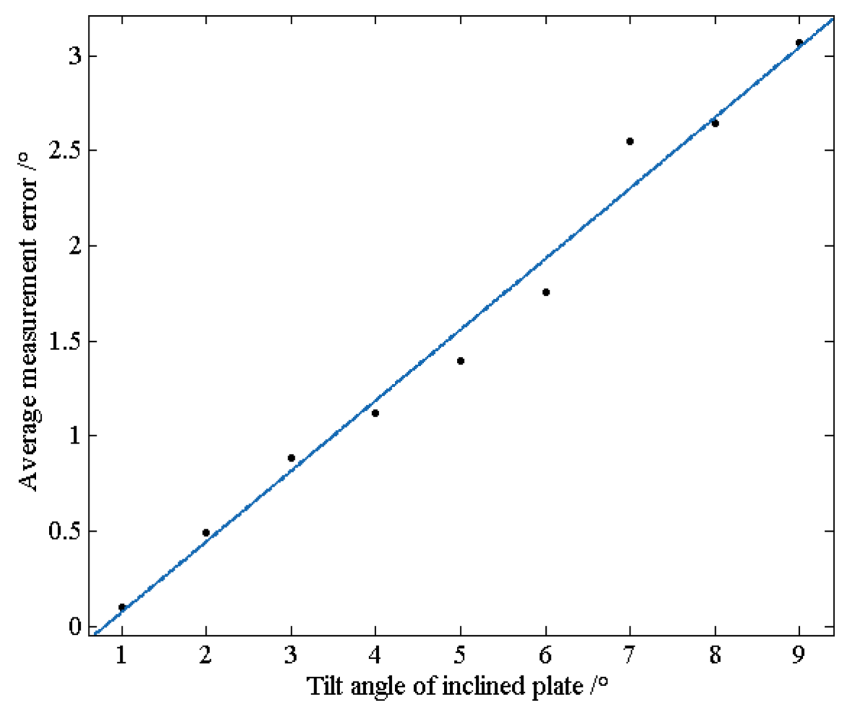

Fig. 9. (Color online) The relation between tilt angle and average error by experiments.

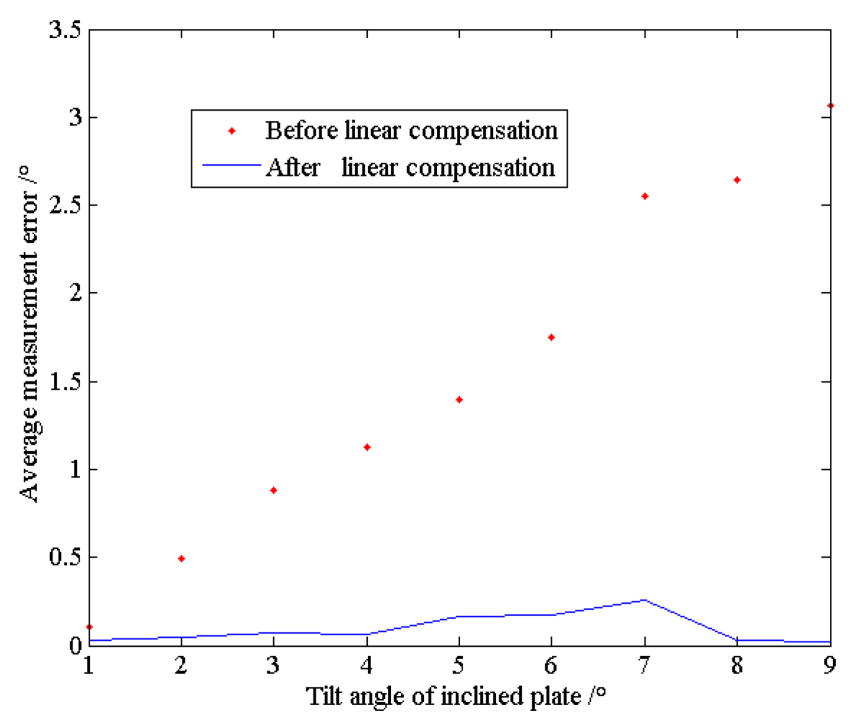

Fig. 10. (Color online) The measured error before and after compensation by experiments. error of five groups is regarded as the final measured error. Furthermore, the relationship between average measurement error and tilt angle of inclined plate is shown as Fig. 9.

It is found that the average measurement error is indeed linear with tilt angle, which verifies the correctness of simulation analysis. The fitting function is as follows:

$$
f(x)=0.3709 * x-0.2981
$$

Where, the variable $x$ represents tilt angle of inclined plate and the variable $f(x)$ stands for the average measurement error. The sum of squares due to error (SSE) is 0.1351 and the coefficient of determination $\left(\mathrm{R}^{2}\right)$ is 0.9839 .

The measurement error before and after linear compensation is shown in Fig. 10. By the experiment, it is shown that the largest measured error after compensation is $0.25^{\circ}$. So, the simulation and experiment analyses are consistent and confirmed by each other.

\section{Results and Discussion}

In this paper, the tilt angle measurement based on arrayed EC sensors has been analyzed. Supported by the careful experimental evidences, it can be found that the proposed method to measure tilt angle is feasible and repeatable. Based on several groups of experiments, the average measured error is approximately linear with the tilt angle, shown as the Fig. 9. Moreover, the largest measured error is $0.25^{\circ}$ after compensation, shown as the Fig. 10.

This research combines the tilt angle measurement with EC testing, which can not only broaden the scope of EC applications, but also overcomes the problem of existing methods (not suitable for the industrial environment).

However, much work should be done to further optimize the proposed application. In this paper, to avoid the magnetic coupling interference, the spacing between sensors is set as the coil's outer diameter. This way will lead to a slightly larger size of the measuring device. Thus, our future work will be focused on the miniaturization.

\section{Acknowledgements}

This work was supported by the Science and Technology Research and Development Program of Shihezi University under Grant [No. 2014ZRKXYQ05].

\section{References}

[1] Y. Yu, G. Tian, X. Li, and A. Simm, Nondestruct Test 
Eval. 28, 195 (2013).

[2] Y. Yu, T. Y, and P. Du, NDT E Int. 48, 16 (2012).

[3] H. Wang, B. Ju, W. Li, and Z. Feng, Sens Actuators Phys. 211, 98 (2014).

[4] L. Banteng, H. Dibo, L. Baoling, Z. Ling, H. Pingjie, and Z. Guangxin, Insight - Non-Destr Test Cond Monit. 56, 375 (2014).

[5] M. Pan, Y. He, G. Tian, D. Chen, and F. Luo, Ndt E Int. 52, 28 (2012).

[6] Y. He, M. Pan, F. Luo, and G. Tian, Ndt E Int. 44, 344 (2011).

[7] I. D. Adewale and G. Y. Tian, IEEE Trans. Magn. 49, 1119 (2013).
[8] W. Yin, P. J. Withers, U. Sharma, and A. J. Peyton, IEEE Trans Instrum Meas. 58, 738 (2009).

[9] T. Bouchala, B. Abdelhadi, and A. Benoudjit, Nondestruct Test Eval. 30, 63 (2015).

[10] M. C. Hsieh, J. Y. Lin, and C. O. Chang, Sensors 16, 1301 (2016).

[11] S. Zhao, Y. Li, E. Zhang, P. Huang, and H. Wei, Rev. Sci. Instrum. 85, 96 (2014).

[12] M. Lian, H. Liu, Y. Wang, Y. Li, X. Sheng, and Y. Ying, Int. J. Automation Technology 10, 977 (2016).

[13] Y. Li, X. Sheng, M. Lian, and Y. Wang, Nondestruct Test Eval. 31, 289 (2016). 